KYUNGPOOK Math. J. 56(2016), 451-464

http://dx.doi.org/10.5666/KMJ.2016.56.2.451

pISSN 1225-6951 eISSN 0454-8124

(C) Kyungpook Mathematical Journal

\title{
A Further Result Related to a Conjecture of R. Brück
}

\author{
NAN LI \\ School of Mathematical Sciences, University of Jinan, Jinan, Shandong, 250022, \\ P. R. China \\ e-mail : nanli32787310@163.com \\ LIANZHONG YANG* \\ School of Mathematics, Shandong University, Jinan, Shandong, 250100, P. R. \\ China \\ e-mail : lzyang@sdu.edu.cn \\ KAI LIU \\ Department of Mathematics, Nanchang University, Nanchang, Jiangxi, 330031, P. \\ R. China \\ e-mail : liukai418@126.com
}

ABSTRACT. In this paper, we investigate the uniqueness problem of a meromorphic function sharing one small function with its differential polynomial, and give a result which is related to a conjecture of R. Brück.

\section{Introduction}

In this paper, meromorphic function means meromorphic in the complex plane. We use the standard notations of Nevanlinna theory, which can be found in [12]. A meromorphic function $a(z)$ is called a small function with respect to $f(z)$ if $T(r, a)=S(r, f)$. We say that two meromorphic functions $f$ and $g$ share a small function $a$ IM (ignoring multiplicities) when $f-a$ and $g-a$ have the same zeros. If $f-a$ and $g-a$ have the same zeros with the same multiplicities, then we say that $f$ and $g$ share $a$ CM (counting multiplicities).

Let $f(z)$ be a meromorphic function. It is known that the hyper order of $f(z)$,

* Corresponding Author.

Received May 9, 2013; accepted April 12, 2016.

2010 Mathematics Subject Classification: 30D35.

Key words and phrases: Meromorphic function, Shared value, Small function.

This work was supported by the NNSF of China (No. 11171013 \& No. 11371225) and the NSF of Shandong Province, P. R. China (N0. ZR2010AM030). 
denoted by $\sigma_{2}(f)$, is defined by

$$
\sigma_{2}(f)=\limsup _{r \rightarrow \infty} \frac{\log \log T(r, f)}{\log r} .
$$

In 1996, R. Brück [1] posed the following conjecture.

Brück Conjecture. Let $f$ be a non-constant entire function such that the hyper order $\sigma_{2}(f)$ of $f$ is not a positive integer and $\sigma_{2}(f)<+\infty$. If $f$ and $f^{\prime}$ share a finite value $a \mathrm{CM}$, then

$$
\frac{f^{\prime}-a}{f-a}=c
$$

where $c$ is a nonzero constant.

In 1998, Gundersen and Yang [3] verified that the Conjecture is true when $f$ is of finite order. In 1999, Yang [10] confirmed that the Conjecture is also true when $f^{\prime}$ is replaced by $f^{(k)}(k \geq 2)$ and $f$ is of finite order. In recent years, many results have been published concerning the above conjecture, see $[2,5,7,8,14,15,16,17,18]$ etc., and Zhang [17] was the first author who considers the case when $f$ is a meromorphic function. We need the following definition.

Definition 1.1. Let $l$ be a non-negative integer or infinite. Denote by $E_{l}(a, f)$ the set of all a-points of $f$ where an a-point of multiplicity $m$ is counted $m$ times if $m \leq l$ and $l+1$ times if $m>l$. If $E_{l}(a, f)=E_{l}(a, g)$, we say that $f$ and $g$ share $(a, l)$.

Remark. It is easy to see that $f$ and $g$ share $(a, l)$ implies that $f$ and $g$ share $(a, p)$ for $0 \leq p \leq l$. Also we note that $f$ and $g$ share the value $a$ IM or CM if and only if $f$ and $g$ share $(a, 0)$ or $(a, \infty)$, respectively. We also use $N_{p}\left(r, \frac{1}{f-a}\right)$ to denote the counting function of the zeros of $f-a$ where a zero of multiplicity $m$ is counted $m$ times if $m \leq p$ and $p$ times if $m>p$.

Lahiri [5] improved the results of Zhang [17] by using the above definition and obtained the following Theorem:

Theorem A. Let $f$ be a non-constant meromorphic function and $k$ be a positive integer. If $f$ and $f^{(k)}$ share $(1,2)$ and

$$
2 \bar{N}(r, f)+N_{2}\left(r, \frac{1}{f^{(k)}}\right)+N_{2}\left(r, \frac{1}{f}\right)<(\lambda+o(1)) T\left(r, f^{(k)}\right),
$$

for $r \in I$, where $0<\lambda<1$ and $I$ is a set of infinite linear measure, then $\frac{f^{(k)}-a}{f-a}=c$ for $c \in \mathbf{C} \backslash\{0\}$.

Let $p$ be a positive integer and $a \in \mathbf{C} \bigcup\{\infty\}$. We use $N_{p)}\left(r, \frac{1}{f-a}\right)$ to denote the counting function of the zeros of $f-a$, whose multiplicities are not greater 
than $p, N_{(p+1}\left(r, \frac{1}{f-a}\right)$ to denote the counting function of the zeros of $f-a$ whose multiplicities are not less than $p+1$, and we use $\bar{N}_{p)}\left(r, \frac{1}{f-a}\right)$ and $\bar{N}_{(p+1}\left(r, \frac{1}{f-a}\right)$ to denote their corresponding reduced counting functions (ignoring multiplicities) respectively. Define

$$
\delta_{p}(a, f)=1-\limsup _{r \rightarrow+\infty} \frac{N_{p}\left(r, \frac{1}{f-a}\right)}{T(r, f)} .
$$

It follows that $\delta_{p}(a, f) \geq \delta(a, f)$.

Let $L(f)=f^{(k)}+a_{k-1} f^{(k-1)}+\cdots+a_{0} f$. Zhang and Yang [16] obtained the following result which improves the results of $[5,8,14,18]$.

Theorem B. Let $f$ be a non-constant meromorphic function, $k \geq 1$ and $l \geq 0$ be integers. Let $a(z)$ be a small function of $f$ such that $a(z) \not \equiv 0, \infty$. Suppose that $f-a$ and $L(f)-a$ share $(0, l)$. Then $f \equiv L(f)$ if one of the following assumptions holds,

(1) $l \geq 2$ and

$$
3 \theta(\infty, f)+\delta_{2+k}(0, f)+\delta_{2}(0, f)+\delta(a, f)>4,
$$

(2) $l=1$ and

$$
\frac{7+k}{2} \theta(\infty, f)+\frac{1}{2} \delta_{1+k}(0, f)+\delta_{2}(0, f)+\delta_{2+k}(0, f)+\delta(a, f)>\frac{k}{2}+5,
$$

(3) $l=0$ and

$$
(2 k+6) \theta(\infty, f)+\delta_{2}(0, f)+2 \delta_{1+k}(0, f)+\delta_{2+k}(0, f)+\theta(0, f)+\delta(a, f)>2 k+10 .
$$

Definition 1.2. Let $p_{0}, p_{1}, \ldots p_{k}$ be non-negative integers. We call

$$
M[f]=f^{p_{0}}\left(f^{\prime}\right)^{p_{1}} \cdots\left(f^{(k)}\right)^{p_{k}}
$$

a differential monomial in $f$ with degree $d_{M}=p_{0}+p_{1}+\cdots+p_{k}$ and weight $\Gamma_{M}=p_{0}+2 p_{1}+\cdots+(k+1) p_{k}$, and

$$
H[f]=\sum_{j=1}^{n} a_{j} M_{j}[f],
$$

where $a_{j}$ are small functions of $f$, is called a differential polynomial in $f$ of degree $d=\max \left\{d_{M_{j}}, 1 \leq j \leq n\right\}$ and weight $\Gamma=\max \left\{\Gamma_{M_{j}}, 1 \leq j \leq n\right\}$, furthermore if $\operatorname{deg}\left(M_{j}\right)=d(j=1,2, \cdots n)$, then $H[f]$ is a homogeneous differential polynomial in $f$ of degree $d$.

In this paper, we improve the above Theorems and obtain the following result.

Theorem 1.3. Let $f$ be a non-constant meromorphic function and $H[f]$ be a nonconstant homogeneous differential polynomial of degree $d$ and weight $\Gamma$ satisfying 
$\Gamma \geq(k+2) d-2$. Let $a(z)$ be a small meromorphic function of $f$ such that $a(z) \not \equiv$ $0, \infty$. Suppose that $f-a$ and $H[f]-a$ share $(0, l)$. Then $\frac{H[f]-a}{f-a}=C$ for some non-zero constant $C$ if one of the following assumptions holds,

(i) $l \geq 2$ and

$$
3 \theta(\infty, f)+d \delta_{2+\Gamma-d}\left(0, f^{d}\right)+\delta_{2}(0, f)+\delta(a, f)>4,
$$

(ii) $l=1$ and

$$
\frac{7+\Gamma-d}{2} \theta(\infty, f)+\frac{d}{2} \delta_{1+\Gamma-d}\left(0, f^{d}\right)+d \delta_{2+\Gamma-d}\left(0, f^{d}\right)+\delta_{2}(0, f)+\delta(a, f)>\frac{\Gamma+9}{2},
$$

(iii) $l=0$ and

$$
\begin{gathered}
(1.3) \quad[2(\Gamma-d)+6] \theta(\infty, f)+\delta_{2}(0, f)+d \delta_{2+\Gamma-d}\left(0, f^{d}\right)+2 d \delta_{1+\Gamma-d}\left(0, f^{d}\right) \\
+\theta(0, f)+\delta(a, f)>2 \Gamma+8 .
\end{gathered}
$$

Especially, when $l=0$, i.e. $f$ and $H$ share $a I M$, if (1.3) holds, then $f \equiv H[f]$.

\section{Some Lemmas}

Lemma 2.1.([11]) Let $f$ be a nonconstant meromorphic function, $k$ be a positive integer. Then

$$
\begin{gathered}
N\left(r, \frac{1}{f^{(k)}}\right) \leq T\left(r, f^{(k)}\right)-T(r, f)+N\left(r, \frac{1}{f}\right)+S(r, f), \\
N\left(r, \frac{1}{f^{(k)}}\right) \leq N\left(r, \frac{1}{f}\right)+k \bar{N}(r, f)+S(r, f) .
\end{gathered}
$$

Suppose that $F$ and $G$ are two non-constant meromorphic functions such that $F$ and $G$ share the value 1 IM. Let $z_{0}$ be a 1-point of $F$ of order $p$, a 1-point of $G$ of order $q$. We denote by $N_{L}\left(r, \frac{1}{F-1}\right)$ the counting function of those 1-points of $F$ where $p>q$, by $N_{E}^{1)}\left(r, \frac{1}{F-1}\right)$ the counting function of those 1-points of $F$ where $p=q=1$, by $N_{E}^{(2}$ the counting function of those 1 -points of $F$ where $p=q \geq 2$; each point in these counting functions is counted only one time. Similarly, we can define $N_{L}\left(r, \frac{1}{G-1}\right), N_{E}^{1)}\left(r, \frac{1}{G-1}\right)$ and $N_{E}^{(2}\left(r, \frac{1}{G-1}\right)$.

Lemma 2.2.([13]) Let $F$ and $G$ are two nonconstant meromorphic functions,

$$
\Delta=\left(\frac{F^{\prime \prime}}{F^{\prime}}-\frac{2 F^{\prime}}{F-1}\right)-\left(\frac{G^{\prime \prime}}{G^{\prime}}-\frac{2 G^{\prime}}{G-1}\right) .
$$


If $F$ and $G$ share 1 IM and $\Delta \not \equiv 0$, then

$$
N_{E}^{1)}\left(r, \frac{1}{F-1}\right) \leq N(r, \Delta)+S(r, F)+S(r, G) .
$$

Lemma 2.3. Let $H[f]$ be a non-constant differential polynomial. Let $z_{0}$ be a pole of $f$ of order $p$ and neither a zero nor a pole of coefficients of $H[f]$. Then $z_{0}$ is a pole of $H[f]$ with order at most $p d+(\Gamma-d)$.

Proof. Let

$$
\begin{gathered}
H[f]=\sum_{j=1}^{n} a_{j} H_{j}[f], \quad H_{j}[f]=f^{p_{0}}\left(f^{\prime}\right)^{p_{1}} \cdots\left(f^{(k)}\right)^{p_{k}}, \\
d_{M_{j}}=p_{0}+p_{1}+\cdots+p_{k}, \quad \Gamma_{M_{j}}=p_{0}+2 p_{1}+\cdots+(k+1) p_{k} .
\end{gathered}
$$

Let $z_{0}$ be a pole of $f$ of order $p$, then $z_{0}$ be a pole of $H_{j}[f]$ of order $p d_{M_{j}}+\left(\Gamma_{M_{j}}-d_{M_{j}}\right)$. Because $d=\max \left\{d_{M_{j}}, 1 \leq j \leq n\right\}, \quad \Gamma=\max \left\{\Gamma_{M_{j}}, 1 \leq j \leq\right.$ $n\}$ and $z_{0}$ neither be a zero nor be a pole of $a_{j}$, then $z_{0}$ is a pole of $\mathrm{H}[\mathrm{f}]$ with order at most $p d+(\Gamma-d)$.

Lemma 2.4. Let $f$ be a transcendental meromorphic function, $H[f]$ is a homogeneous differential polynomial in $f$ of degree $d$ and weight $\Gamma$. If $H[f] \not \equiv 0$, we have

$$
\begin{gathered}
N\left(r, \frac{1}{H}\right) \leq T(r, H)-d T(r, f)+d N\left(r, \frac{1}{f}\right)+S(r, f) . \\
N\left(r, \frac{1}{H}\right) \leq(\Gamma-d) \bar{N}(r, f)+d N\left(r, \frac{1}{f}\right)+S(r, f) .
\end{gathered}
$$

Proof. By the first fundamental theorem and the lemma of logarithmic derivatives, we have

$$
\begin{aligned}
N\left(r, \frac{1}{H}\right) & =T(r, H)-m\left(r, \frac{1}{H}\right)+O(1) \\
& \leq T(r, H)-\left(m\left(r, \frac{1}{f^{d}}\right)-m\left(r, \frac{H}{f^{d}}\right)\right)+O(1) \\
& =T(r, H)-\left(T\left(r, \frac{1}{f^{d}}\right)-N\left(r, \frac{1}{f^{d}}\right)\right)+S(r, f) \\
& =T(r, H)-d T(r, f)+d N\left(r, \frac{1}{f}\right)+S(r, f) .
\end{aligned}
$$


This proves (2.5). From Lemma 3, we have

$$
\begin{aligned}
T(r, H) & =m(r, H)+N(r, H) \\
& \leq m\left(r, \frac{H}{f^{d}}\right)+m\left(r, f^{d}\right)+N(r, H) \\
& \leq d m(r, f)+d N(r, f)+(\Gamma-d) \bar{N}(r, f)+S(r, f) \\
& =d T(r, f)+(\Gamma-d) \bar{N}(r, f)+S(r, f) .
\end{aligned}
$$

Combining with (2.7), we obtain (2.6).

Lemma 2.5. Let $f$ be a non-constant meromorphic function, $H[f]$ is a homogeneous differential polynomial in $f$ of degree $d$ and weight $\Gamma$, and let $p$ be a positive integer. If $H[f] \not \equiv 0$ and $\Gamma \geq(k+2) d-(p+1)$, we have

$$
N_{p}\left(r, \frac{1}{H}\right) \leq T(r, H)-d T(r, f)+N_{p+\Gamma-d}\left(r, \frac{1}{f^{d}}\right)+S(r, f),
$$

$$
N_{p}\left(r, \frac{1}{H}\right) \leq(\Gamma-d) \bar{N}(r, f)+N_{p+\Gamma-d}\left(r, \frac{1}{f^{d}}\right)+S(r, f)
$$

Proof. From (2.6), we have

$$
\begin{aligned}
N_{p}\left(r, \frac{1}{H}\right)+\sum_{j=p+1}^{\infty} \bar{N}_{(j}\left(r, \frac{1}{H}\right) \leq & (\Gamma-d) \bar{N}(r, f)+N_{p+\Gamma-d}\left(r, \frac{1}{f^{d}}\right) \\
& +\sum_{j=p+\Gamma-d+1}^{\infty} \bar{N}_{(j}\left(r, \frac{1}{f^{d}}\right)+S(r, f) .
\end{aligned}
$$

Since $\Gamma \geq(k+2) d-(p+1)$, then we have

$$
\begin{aligned}
N_{p}\left(r, \frac{1}{H}\right) \leq & (\Gamma-d) \bar{N}(r, f)+N_{p+\Gamma-d}\left(r, \frac{1}{f^{d}}\right)+\sum_{j=p+\Gamma-d+1}^{\infty} \bar{N}_{(j}\left(r, \frac{1}{f^{d}}\right) \\
& -\sum_{j=p+1}^{\infty} \bar{N}_{(j}\left(r, \frac{1}{H}\right)+S(r, f) \\
\leq & (\Gamma-d) \bar{N}(r, f)+N_{p+\Gamma-d}\left(r, \frac{1}{f^{d}}\right)+S(r, f) .
\end{aligned}
$$


Thus (2.9) holds. By the same arguments as above, we obtain (2.8) from (2.5).

\section{Proof of Theorem 1.3}

Let $F=\frac{H[f]}{a}, G=\frac{f}{a}$. From the conditions of Theorem 1.3, we know that $F$ and $G$ share $(1, l)$ except the zeros and poles of a(z). From the proof of Lemma 2.4, we have

$$
T(r, F)=O(T(r, f))+S(r, f), \quad T(r, G)=T(r, f)+S(r, f) .
$$

It is obvious that $f$ is a transcendental meromorphic function. Let $\Delta$ be defined by (2.3). We distinguish two cases.

Case 1. $\Delta \equiv 0$. Integrating $(2.3)$, yields

$$
\frac{1}{G-1}=\frac{C}{F-1}+D
$$

where $C$ and $D$ are constants and $C \neq 0$. If there exists a pole $z_{0}$ of $f$ with multiplicity $p$ which is not zero or pole of $a$, then $z_{0}$ is a pole of $F$ with multiplicity $p d+(\Gamma-d)$, a pole of $G$ with multiplicity $p$. This contradicts (3.2) as $H$ contains at least one derivative. Therefore, we have

$$
\bar{N}(r, F)=\bar{N}(r, G)=\bar{N}(r, f)=S(r, f) .
$$

(3.2) also shows that $F$ and $G$ share the value $1 \mathrm{CM}$.

Next, we will prove $D=0$.

Suppose $D \neq 0$, then we have

$$
\frac{1}{G-1}=\frac{D\left(F-1+\frac{C}{D}\right)}{F-1}
$$

So, we have

$$
\bar{N}\left(r, \frac{1}{D\left(F-1+\frac{C}{D}\right)}\right)=\bar{N}\left(r, \frac{G-1}{F-1}\right)=S(r, f) .
$$

Subcase 1.1. If $\frac{C}{D} \neq 1$, then by using (3.3), (3.5) and the second fundamental theorem, we have

$$
\begin{aligned}
T(r, F) & \leq \bar{N}(r, F)+\bar{N}\left(r, \frac{1}{F}\right)+\bar{N}\left(r, \frac{1}{F-1+\frac{C}{D}}\right)+S(r, F) \\
& \leq \bar{N}\left(r, \frac{1}{F}\right)+S(r, F) \leq(1+o(1)) T(r, F)
\end{aligned}
$$


This gives that

$$
T(r, F)=\bar{N}\left(r, \frac{1}{F}\right)+S(r, F)=N_{1}\left(r, \frac{1}{F}\right)+S(r, F) .
$$

So we have

$$
T(r, H)=N\left(r, \frac{1}{H}\right)+S(r, f)=N_{1}\left(r, \frac{1}{H}\right)+S(r, f) .
$$

Let $p=1$, then from assumption we have

$$
\Gamma \geq(k+2) d-2=(k+2) d-(p+1) .
$$

Thus from (2.8) in Lemma 2.5, we get

$$
T(r, H)=N_{1}\left(r, \frac{1}{H}\right)+S(r, f) \leq T(r, H)-d T(r, f)+N_{1+\Gamma-d}\left(r, \frac{1}{f^{d}}\right)+S(r, f) .
$$

So we have

$$
d T(r, f) \leq N_{1+\Gamma-d}\left(r, \frac{1}{f^{d}}\right)+S(r, f) .
$$

This gives that

$$
d T(r, f)=N_{1+\Gamma-d}\left(r, \frac{1}{f^{d}}\right)+S(r, f) .
$$

So we have

$$
\delta_{2+\Gamma-d}\left(0, f^{d}\right)=\delta_{1+\Gamma-d}\left(0, f^{d}\right)=0 .
$$

Since (3.3), we get

$$
\theta(\infty, f)=1
$$

Subcase 1.1.1. $l \geq 2$.

From $\delta_{2}(0, f)+\delta(a, f)>1$ and the definition of deficiency, we have

$$
T(r, f)>N_{2}\left(r, \frac{1}{f}\right)+N\left(r, \frac{1}{f-a}\right) .
$$

Using the second fundamental theorem of Nevanlinna and (3.3), we have

$$
\begin{aligned}
T(r, f) & \leq \bar{N}\left(r, \frac{1}{f}\right)+\bar{N}\left(r, \frac{1}{f-a}\right)+\bar{N}(r, f)+S(r, f) \\
& =\bar{N}(r, 1 f)+\bar{N}\left(r, \frac{1}{f-a}\right)+S(r, f) .
\end{aligned}
$$


Combining (3.7) with (3.8), we have

$N_{2}\left(r, \frac{1}{f}\right)+N\left(r, \frac{1}{f-a}\right)<T(r, f) \leq \bar{N}\left(r, \frac{1}{f}\right)+\bar{N}\left(r, \frac{1}{f-a}\right)+S(r, f)$.

So we have

$$
N_{2}\left(r, \frac{1}{f}\right)=S(r, f), N\left(r, \frac{1}{f-a}\right)=S(r, f) .
$$

This gives that

$$
\bar{N}\left(r, \frac{1}{f}\right)=S(r, f), \bar{N}\left(r, \frac{1}{f-a}\right)=S(r, f) .
$$

From (3.8), we get a contradiction.

Subcase 1.1.2. $l=1$.

When $d \geq 2$, by using (1.2) and the definition of deficiency, we get a contradiction. When $d=1$, using the similar method in subcase 1.1.1, we get a contradiction.

Subcase 1.1.3. $l=0$.

By using (1.3) and the definition of deficiency, we get a contradiction.

Subcase 1.2. If $\frac{C}{D}=1$, then from (3.4), we have

$$
\frac{1}{G-1} \equiv C \frac{F}{F-1}
$$

This gives us that

$$
\left(G-1-\frac{1}{C}\right) F \equiv-\frac{1}{C}
$$

Using that $F=\frac{H}{a}$ and $G=\frac{f}{a}$, we get

$$
f-a\left(1+\frac{1}{C}\right) \equiv-\frac{a^{2}}{C} \cdot \frac{1}{H} .
$$

Using (3.3) (3.9), Lemma 2.3 and the first fundamental theorem, we get

$$
\begin{aligned}
(d+1) T(r, f) & =T\left(r, \frac{1}{f^{d}\left(f-\left(1+\frac{1}{C}\right) a\right)}\right)+O(1) \\
& =T\left(r,-\frac{C H}{f^{d} a^{2}}\right)+O(1) \\
& =N\left(r, \frac{H}{f^{d}}\right)+S(r, f) \\
& \leq d N\left(r, \frac{1}{f}\right)+S(r, f) \\
& \leq(d+o(1)) T(r, f),
\end{aligned}
$$


which is a contradiction, hence $\mathrm{D}=0$. This gives from (3.2) that

$$
\frac{F-1}{G-1} \equiv C \text {. }
$$

So we get $\frac{H[f]-a}{f-a}=C(C \neq 0)$.

Next, we will prove $C=1$ when $l=0$.

Suppose $C \neq 1$, then we have

$$
G \equiv \frac{1}{C}(F-1+C)
$$

and

$$
N\left(r, \frac{1}{G}\right)=N\left(r, \frac{1}{F-1+C}\right) .
$$

By the second fundamental theorem and (3.3) (3.10), we have

$$
\begin{aligned}
T(r, F) & \leq \bar{N}(r, F)+\bar{N}\left(r, \frac{1}{F}\right)+\bar{N}\left(r, \frac{1}{F-1+C}\right)+S(r, f) \\
& \leq \bar{N}\left(r, \frac{1}{F}\right)+\bar{N}\left(r, \frac{1}{G}\right)+S(r, f) \\
& =N_{1}\left(r, \frac{1}{F}\right)+\bar{N}\left(r, \frac{1}{G}\right) .
\end{aligned}
$$

By Lemma 2.5 for $p=1$, we have

$$
d T(r, f) \leq N_{1+\Gamma-d}\left(r, \frac{1}{f^{d}}\right)+\bar{N}\left(r, \frac{1}{f}\right)+S(r, f) .
$$

From the above formula and the definition of deficiency, we have

$$
d \delta_{1+\Gamma-d}\left(0, f^{d}\right)+\theta(0, f) \leq 1 .
$$

So we have

$$
d \delta_{2+\Gamma-d}\left(0, f^{d}\right)+\delta_{2}(0, f) \leq 1, d \delta_{1+\Gamma-d}\left(0, f^{d}\right) \leq 1 .
$$

Combining (3.11) (3.12) (3.6) with the assumptions of Theorem 1.3, we get a contradiction.

So $C=1$ and $G \equiv F$, i.e. $f \equiv H[f]$.

This is just the conclusion of this theorem. 
Case 2. $\Delta \not \equiv 0$.

By a similar method that used in the proof of Theorem B[16], we get

$$
\begin{aligned}
T(r, F)+T(r, G) & \leq \bar{N}\left(r, \frac{1}{F}\right)+\bar{N}(r, F)+\bar{N}\left(r, \frac{1}{F-1}\right) \\
& +\bar{N}\left(r, \frac{1}{G}\right)+\bar{N}(r, G)+\bar{N}\left(r, \frac{1}{G-1}\right) \\
& -N_{0}\left(r, \frac{1}{F^{\prime}}\right)-N_{0}\left(r, \frac{1}{G^{\prime}}\right)+S(r, f)
\end{aligned}
$$

and

$$
\begin{aligned}
\bar{N}\left(r, \frac{1}{F-1}\right)+ & \bar{N}\left(r, \frac{1}{G-1}\right) \leq \bar{N}_{(2}\left(r, \frac{1}{F}\right)+\bar{N}_{(2}\left(r, \frac{1}{G}\right) \\
& +\bar{N}(r, G)+3 N_{L}\left(r, \frac{1}{F-1}\right)+3 N_{L}\left(r, \frac{1}{G-1}\right)+N_{E}^{1)}\left(r, \frac{1}{F-1}\right) \\
& +2 N_{E}^{(2}\left(r, \frac{1}{G-1}\right)+N_{0}\left(r, \frac{1}{F^{\prime}}\right)+N_{0}\left(r, \frac{1}{G^{\prime}}\right)+S(r, f) .
\end{aligned}
$$

Subcase 2.1. $l \geq 2$. It is easy to see that

$$
\begin{aligned}
3 N_{L}\left(r, \frac{1}{F-1}\right)+3 N_{L}\left(r, \frac{1}{G-1}\right) & +2 N_{E}^{(2}\left(r, \frac{1}{G-1}\right)+N_{E}^{1)}\left(r, \frac{1}{F-1}\right) \\
& \leq N\left(r, \frac{1}{G-1}\right)+S(r, f) .
\end{aligned}
$$

From (3.13) (3.14) and (3.15), we have

$$
\begin{aligned}
T(r, F)+T(r, G) & \leq 3 \bar{N}(r, G)+N_{2}\left(r, \frac{1}{F}\right) \\
& +N_{2}\left(r, \frac{1}{G}\right)+N\left(r, \frac{1}{G-1}\right)+S(r, f) .
\end{aligned}
$$

Noting that

$$
N_{2}\left(r, \frac{1}{F}\right)=N_{2}\left(r, \frac{a}{H}\right) \leq N_{2}\left(r, \frac{1}{H}\right)+S(r, f) .
$$

Let $p=2$, then from assumption we have

$$
\Gamma \geq(k+2) d-2>(k+2) d-(p+1) .
$$

Thus, from (2.8) in Lemma 2.5 we obtain that

$$
\begin{aligned}
T(r, H)+T(r, f) & \leq 3 \bar{N}(r, f)+T(r, H)-d T(r, f)+N_{2+\Gamma-d}\left(r, \frac{1}{f^{d}}\right) \\
& +N_{2}\left(r, \frac{1}{f}\right)+T(r, f)-m\left(r, \frac{1}{f-a}\right)+S(r, f) .
\end{aligned}
$$


So we have

$d T(r, f) \leq 3 \bar{N}(r, f)+N_{2+\Gamma-d}\left(r, \frac{1}{f^{d}}\right)+N_{2}\left(r, \frac{1}{f}\right)-m\left(r, \frac{1}{f-a}\right)+S(r, f)$.

This gives that

$$
3 \theta(\infty, f)+d \delta_{2+\Gamma-d}\left(0, f^{d}\right)+\delta_{2}(0, f)+\delta(a, f) \leq 4 .
$$

Which contradicts the assumption (1.1) of Theorem 1.3.

Subcase 2.2. $l=1$. Noting that

$$
\begin{aligned}
2 N_{L}\left(r, \frac{1}{F-1}\right)+3 N_{L}\left(r, \frac{1}{G-1}\right) & +2 N_{E}^{(2}\left(r, \frac{1}{G-1}\right)+N_{E}^{1)}\left(r, \frac{1}{F-1}\right) \\
\leq & N\left(r, \frac{1}{G-1}\right)+S(r, f)
\end{aligned}
$$

and

$$
\begin{aligned}
N_{L}\left(r, \frac{1}{F-1}\right) & \leq \frac{1}{2} N\left(r, \frac{F}{F^{\prime}}\right) \leq \frac{1}{2} T\left(r, \frac{F}{F^{\prime}}\right)=\frac{1}{2} T\left(r, \frac{F^{\prime}}{F}\right)+O(1) \\
& \leq \frac{1}{2} N\left(r, \frac{F^{\prime}}{F}\right)+S(r, f) \\
& \leq \frac{1}{2}\left(\bar{N}\left(r, \frac{1}{F}\right)+\bar{N}(r, F)\right)+S(r, f) \\
& \leq \frac{1}{2}\left(\bar{N}\left(r, \frac{1}{H}\right)+\bar{N}(r, f)\right)+S(r, f) \\
& \leq \frac{1}{2}\left[(\Gamma-d+1) \bar{N}(r, f)+N_{1+\Gamma-d}\left(r, \frac{1}{f^{d}}\right)\right]+S(r, f) .
\end{aligned}
$$

Using the same method as Subcase 2.1, we get

$$
\begin{aligned}
d T(r, f) \leq & \frac{\Gamma-d+7}{2} \bar{N}(r, f)+N_{2+\Gamma-d}\left(r, \frac{1}{f^{d}}\right)+\frac{1}{2} N_{1+\Gamma-d}\left(r, \frac{1}{f^{d}}\right) \\
& +N_{2}\left(r, \frac{1}{f}\right)-m\left(r, \frac{1}{f-a}\right)+S(r, f) .
\end{aligned}
$$

Which contradicts with (1.2) of Theorem 1.3.

Subcase 2.3. $l=0$. Noting that

$$
\begin{aligned}
N_{L}\left(r, \frac{1}{F-1}\right)+2 N_{L}\left(r, \frac{1}{G-1}\right) & +2 N_{E}^{(2}\left(r, \frac{1}{G-1}\right)+N_{E}^{1)}\left(r, \frac{1}{F-1}\right) \\
\leq & N\left(r, \frac{1}{G-1}\right)+S(r, f) .
\end{aligned}
$$


From Lemma 2.5, we have

$$
\begin{aligned}
N_{L}\left(r, \frac{1}{F-1}\right) & \leq N\left(r, \frac{F}{F^{\prime}}\right) \\
& \leq N\left(r, \frac{F^{\prime}}{F}\right)+S(r, f) \\
& \leq\left(\bar{N}\left(r, \frac{1}{F}\right)+\bar{N}(r, F)\right)+S(r, f) \\
& \leq\left(\bar{N}\left(r, \frac{1}{H}\right)+\bar{N}(r, f)\right)+S(r, f) \\
& \leq N_{1+\Gamma-d}\left(r, \frac{1}{f^{d}}\right)+(\Gamma-d+1) \bar{N}(r, f)+S(r, f)
\end{aligned}
$$

So we have

$$
\begin{aligned}
2 N_{L}\left(r, \frac{1}{F-1}\right)+N_{L}\left(r, \frac{1}{G-1}\right) & \leq 2 N_{1+\Gamma-d}\left(r, \frac{1}{f^{d}}\right)+2(\Gamma-d+1) \bar{N}(r, f) \\
& +\bar{N}\left(r, \frac{1}{f}\right)+\bar{N}(r, f)+S(r, f) .
\end{aligned}
$$

Combining (3.13) (3.14) (3.16) with (3.17), we have

$$
\begin{aligned}
T(r, H)+T(r, f) \leq & N_{2}\left(r, \frac{1}{H}\right)+N_{2}\left(r, \frac{1}{f}\right)+3 \bar{N}(r, f)+N\left(r, \frac{1}{f-a}\right) \\
& +2 N_{1+\Gamma-d}\left(r, \frac{1}{f^{d}}\right)+2(\Gamma-d+1) \bar{N}(r, f) \\
& +\bar{N}\left(r, \frac{1}{f}\right)+\bar{N}(r, f)+S(r, f) .
\end{aligned}
$$

From (2.8), we have

$$
N_{2}\left(r, \frac{1}{H}\right) \leq T(r, H)-d T(r, f)+N_{2+\Gamma-d}\left(r, \frac{1}{f^{d}}\right)+S(r, f) .
$$

Substituting this into (3.18), we have

$$
\begin{aligned}
d T(r, f) & \leq N_{2}\left(r, \frac{1}{f}\right)+2(\Gamma-d+3) \bar{N}(r, f)+\bar{N}\left(r, \frac{1}{f}\right) \\
& +2 N_{1+\Gamma-d}\left(r, \frac{1}{f^{d}}\right)+N_{2+\Gamma-d}\left(r, \frac{1}{f^{d}}\right)-m\left(r, \frac{1}{f-a}\right)+S(r, f) .
\end{aligned}
$$

So we have

$$
\begin{aligned}
& \delta_{2}(0, f)+\theta(0, f)+2(\Gamma-d+3) \theta(\infty, f)+d \delta_{2+\Gamma-d}\left(0, f^{d}\right) \\
& +2 d \delta_{1+\Gamma-d}\left(0, f^{d}\right)+\delta(a, f) \leq 2 \Gamma+8
\end{aligned}
$$


Which contradicts the assumption of Theorem 1.3.

Now the proof has been completed.

\section{References}

[1] R. Bruck, On entire functions that share one value CM with their derivative, Results in Math, 30(1996), 21-24.

[2] Z. X. Chen and K. H. Shon, On conjecture of R. Brück concerning the entire function sharing one value CM with its derivative, Taiwanese J. Math., 8(2004), 235-244.

[3] G. G. Gundersen and L. Z. Yang, Entire functions that share one value with one or two of their derivatives, J. Math. Anal. Appl., 223(1998), 88-95.

[4] W. K. Hayman, The local growth of power series: a survey of the Wiman-Valiron method, Canad. Math. Bull., 17(1974), 317-358.

[5] I. Lahiri, Uniqueness of a meromorphic function and its derivative, J. Inequal. Pure Appl. Math, 5(1)(2004), Art.20.

[6] I. Laine, Nevanlinna Theory and Complex Differential Equation, Walter de Gruyter, Berlin-New York, (1993).

[7] N. Li and L. Z. Yang, Meromorphic Function that Shares One Small Function with its Differential Polynomial, KYUNGPOOK Math. J., 50(2010), 447-454.

[8] L. P. Liu and Y. X. Gu, Uniqueness of meromorphic functions that share one small function with their derivatives, Kodai Math. J., 27(2004), 272-279.

[9] L. Yang, Value Distribution Theory, Springer-Verlag, Berlin, 1993.

[10] L. Z. Yang, Solution of a differential equation and its applications, Kodai Math. J, 22(1999), 458-464.

[11] H. X. Yi, Uniqueness of meromorphic functions and a question of C.C.Yang, Complex Variables, 14(1990), 169-176.

[12] H. X. Yi and C. C. Yang, Uniqueness theory of meromorphic functions, Science Press, Beijing, (1995).

[13] H. X. Yi, Uniqueness theorems for meromorphic functions whose $n$-th derivatives share the same 1-points, Complex Variables, 34(1997), 421-436.

[14] K. W. Yu, On entire and meromorphic functions that share small functions with their derivatives, J. Ineq. Pure and Appl. Math., 4(1) Art. 21, 2003.

[15] J. L. Zhang and L. Z.Yang, Some results related to a conjecture of R. Brück concerning meromorphic functions sharing one small function with their derivatives, Annales Academiae Scientiarum Fennicae Mathematica, 32(1)(2007), 141-149.

[16] J. L. Zhang and L. Z.Yang, Some results related to a conjecture of R.Bruck, J. Ineq. Pure Appl. Math, 8(1)(2007), Art. 18.

[17] Q. C. Zhang, The uniqueness of meromorphic functions with their derivatives, Kodai Math. J., 21(2)(1998), 179-184.

[18] Q. C. Zhang, Meromorphic function that share one small function with its derivative, J. Ineq. Pure Appl. Math., 6(4)(2005), Art. 116. 